\title{
El salto a la nueva extrema derecha: una aproxima- ción a los votantes de Plataforma per Catalunya
}

\author{
Aitor HERNÁNDEZ-CARR \\ Institut de Govern i Polítiques Publiques-Universitat Autònoma de Barcelona \\ aitor.carr@uab.cat
}

Recibido: 09-01-2012

Aceptado: 29-01-2013

\begin{abstract}
Resumen
El partido anti-inmigración Plataforma per Catalunya ha experimentado un notable crecimiento electoral a lo largo de los últimos años. A partir de diferentes entrevistas a votantes del partido, el texto trata de descifrar los motivos por los que electores que no guardaban ningún tipo de relación con el ámbito político de la extrema derecha tradicional han pasado a votar a un partido que es socialmente etiquetado como tal. Tres son los grandes ejes explicativos que se desarrollan a lo largo del texto. En primer lugar, diferentes elementos de la trayectoria electoral y valores ideológicos de sus votantes muestran que el partido se está beneficiando de forma directa de procesos como el desalineamiento electoral y la desafección política. Asimismo, estaría consiguiendo posicionarse como una oferta política legítima de cara a vehicular parte del voto de protesta contra los partidos políticos tradicionales. En segundo lugar, el partido ha desarrollado un discurso en relación al "eje nacional" (CatalunyaEspaña) que le estaría permitiendo distinguirse, hasta cierto punto, de la estigmatizada extrema derecha ultra-españolista. Finalmente, el partido ofrece una "respuesta autoritaria" a un electorado que considera que, a raíz de la "masiva” llegada de inmigración, la sociedad ha entrado en una dinámica negativa que amenaza su bienestar en múltiples ámbitos.
\end{abstract}

Palabras clave Nueva extrema derecha, extrema derecha tradicional, xenofobia, desalineamiento electoral, desafección política

\section{Voting for the extreme right: an analysis of Platform for Catalonia's voters}

\begin{abstract}
The anti-immigration party Platform for Catalonia has experienced a relevant electoral growth over the past few years. Through a series of interviews with party voters, the text tries to understand the reasons why voters that did not have any relation with the traditional extreme right have come to vote for a party that is socially labeled as such. Three main explanatory factors are developed throughout the text. First, different elements of the electoral path and ideological values of its voters show that the party is benefiting directly from processes such as electoral misalignment and political disaffection and has been able to position itself as a legitimate instrument to canalize part of the protest vote against traditional political parties. Second, the party is developing a stance regarding the "national axis" (Catalonia-Spain) that may be a key feature in its goal of distinguishing itself from the stigmatized ultra-nationalist extreme right. Finally, the party offers an "authoritarian" answer to an electorate that believes that, due to "massive" immigration, society has slipped into a negative path that threatens their personal well-being.
\end{abstract}


Key words: New extreme right, traditional extreme right, xenophobia, electoral misalignment, political disaffection.

\section{Referencia normalizada}

Hernández-Carr, A. (2013). "El salto a la nueva extrema derecha: una aproximación a los votantes de Plataforma per Catalunya”. Política y Sociedad, Vol.50 Núm. 2 601-627

Sumario: Introducción. 1.De la desalineación electoral al voto de protesta. 2.Entre el ultraespañolismo y el nacionalismo catalán: en busca de la tercera vía. 3.Inmigración: el trampolín hacia la nueva extrema derecha. 4.Conclusiones y futuros interrogantes. Bibliografía

\section{Introducción}

España es uno de los pocos países europeos en que no se ha producido la emergencia de una formación cuyo discurso esté basado en una oposición frontal a la inmigración extranjera y en la crítica a los partidos políticos tradicionales. Una circunstancia que contrasta fuertemente con la irrupción a partir de la década de los ochenta en gran parte del continente europeo de una serie de partidos que han sido definidos como una "nueva extrema derecha" o "derecha radical populista", . Estos partidos han conseguido establecer una base electoral amplia y relativamente estable que les ha permitido tener un creciente protagonismo en el escenario político europeo.

Plataforma per Catalunya es el primer partido del estado español que, a partir de un programa político similar al de la nueva extrema derecha europea, ha conseguido atraer a un creciente número de votantes y evitar la marginalidad electoral que ha acompañado a los partidos de la extrema derecha española. El partido, fundado en el 2002, ha pasado de ser una formación presente exclusivamente en la política municipal y con un apoyo concentrado en ciertos municipios de Catalunya, a ser la primera fuerza política extra-parlamentaria catalana. En este sentido, ha conseguido incrementar de forma notable su base electoral hasta llegar a los 75,000 votos obtenidos en las elecciones autonómicas catalanas del 2010 e irrumpir con fuerza en algunos de los grandes núcleos urbanos del área metropolitana de Barcelona en las municipales del 2011.

${ }^{1}$ Nos estamos refiriendo a partidos como el Frente Nacional francés, el Partido de la Libertad austriaco o los Partidos del Progreso escandinavos. Para una completa clasificación y caracterización de esta familia de partidos véase el trabajo de Norris (2005) y Mudde (2007). 
La formación, liderada por una persona procedente de la extrema derecha tradicional, Josep Anglada ${ }^{2}$, ha tratado de dejar atrás aquellos elementos de la tradición política de la extrema derecha española que pueden lastrar sus posibilidades de éxito electoral y centrarse de forma exclusiva en los discursos y formas de movilización que han resultado electoralmente eficaces para la nueva extrema derecha europea. En este sentido, la estrategia política de PxC puede ser comprendida como el intento de distinguirse de la estigmatizada extrema derecha española y acercarse a las exitosas formaciones de derecha radical populista europeas (Hernández-Carr, 2011a). Consideramos que Plataforma per Catalunya puede ser definido como un partido de "derecha radical populista" a partir de la propuesta hecha por Mudde (2007) para conceptualizar a la nueva "oleada" de partidos que han emergido a partir de los años ochenta. Dicho autor considera que los principales ejes ideológicos de dichos partidos son el nativismo, el populismo y el autoritarismo, y que estos partidos constituyen una familia de partidos propia y diferenciada de la extrema derecha (Mudde, 2007: 19).

La comprensión de la emergencia de los partidos de extrema derecha ha generado diferentes modelos o teorías explicativas. De forma resumida pueden señalarse tres aproximaciones analíticas que han enfatizado diferentes aspectos del fenómeno. En primer lugar, encontramos las teorías de "primera generación" centradas en el análisis sociológico de las bases sociales de estos partidos. A partir de textos ya clásicos como el de Lipset (1970), estos trabajos centran su análisis en la demanda electoral que posibilitaría la emergencia de estos partidos (Evans, 2005). En segundo lugar encontramos las teorías de "segunda generación", más centradas en el posicionamiento político e ideológico de estos partidos y su emplazamiento en el conjunto del tablero político (Kitschelt, 1995). Finalmente, una serie de aproximaciones de "tercera generación" han tratado de articular varios planos de interpretación. Trabajos como el de Norris (2005), cuyo análisis incluye tanto las variables de la demanda electoral como de la oferta política, así como variables institucionales que conformarían la "estructura de oportunidades políticas" dentro de la cual debe comprenderse la "agencia estratégica" de estos partidos.

Partiendo de la base que es necesario tener en cuenta las diferentes perspectivas analíticas que acabamos de apuntar, en el presente texto hemos optado por profundizar en la comprensión de las bases electorales de Plataforma per Catalunya. En este sentido, el texto indaga en y presenta los motivos del apoyo electoral que una franja del electorado catalán ha otorgado a este partido. Para ello nos basaremos principalmente en los resultados de una serie de entrevistas realizadas a votantes de PxC a lo largo de las semanas previas a las elecciones autonómicas catalanas del 2010. La práctica totalidad de los entrevistados no había votado anteriormente al partido y no tenía conexión alguna con el ámbito político de la extrema derecha

2 Josep Anglada fue miembro del partido neo-franquista Fuerza Nueva. Sobre su trayectoria política véanse los trabajos de Casals (2006) y Erra y Serra (2008). 
tradicional. En este sentido, el texto es una oportunidad para entender por qué individuos que nunca han tenido una relación con este ámbito político pasan a apoyar a un partido que es socialmente etiquetado como "de extrema derecha”. Este "salto" a la nueva extrema derecha es la clave de la amplia base electoral conseguida por las formaciones de derecha radical populista en Europa (Faniel, 2000).

La decisión de aproximarse al electorado de PxC a partir de una metodología cualitativa basada en entrevistas a votantes del partido se debe a varios motivos. En primer lugar, el escaso porcentaje de voto obtenido por el partido, junto al gran volumen de voto oculto que presentan este tipo de formaciones, ha hecho que, hasta el momento, las encuestas no recojan una muestra de votantes del partido lo suficientemente nutrida y que apenas exista información sobre este electorado ${ }^{3}$. En segundo lugar, más allá de la falta de datos de encuesta, se ha escogido esta metodología porque se considera que, de cara a comprender la atracción experimentada por los electores, además de las características “objetivas” de éstos hay que incorporar al análisis sus creencias y percepciones "subjetivas" (Eatwell, 2003). Asimismo, consideramos que es un error entender este voto como la simple expresión de un "extremismo irracional". Los votantes realizan un análisis de diferentes factores sociales y políticos y, en función de dicho análisis, argumentan la oportunidad o necesidad de votar a la nueva extrema derecha. En este sentido, tratar de hacer "aflorar" los ejes de su argumentación es central para comprender adecuadamente este fenómeno (Swyngedouw, 2001).

Las entrevistas se realizaron en las semanas previas a las elecciones autonómicas catalanas del 2010 y en total se entrevistó por vía telefónica a 40 personas y otras 20 accedieron a contestar un cuestionario confeccionado a partir del guión de las entrevistas. El contacto inicial se realizó a través de Internet, concretamente a través de la red social Facebook, y también se usó la técnica de la bola de nieve para conseguir nuevos entrevistados. El uso de internet y las redes sociales como vía de acceso a los entrevistados implica inevitablemente un filtraje hacia un determinado segmento de la población (especialmente por la mayor juventud de los usuarios de Internet) ${ }^{4}$. Sin embargo, esta vía de acceso ha permitido el contacto directo con un gran número de potenciales votantes del partido que difícilmente hubiese sido posible realizar de otra forma. En este sentido, cabe destacar que Internet y, especialmente, las redes sociales se están convirtiendo en un ámbito central tanto en la relación de los partidos de nueva extrema derecha con sus potenciales votantes como en la investigación sobre este fenómeno (Littler, 2011).

${ }^{3}$ La única excepción es el trabajo de Pardos-Prado y Molins (2010) en que se realiza un análisis agregado del voto a PxC en las elecciones municipales del 2003 y 2007.

${ }^{4}$ Los participantes en el trabajo de campo tienen una media de edad de 34,6 años y el porcentaje de hombres es del $60 \%$ y de mujeres del $40 \%$. A nivel laboral, un $42 \%$ estaba desocupado, otro $42 \%$ ocupado, un $13 \%$ eran estudiantes y un $3 \%$ pensionistas. 
La información obtenida a partir de las entrevistas se ha combinado con tres fuentes de información complementarias. En primer lugar, dado que anteriormente se ha analizado detalladamente el discurso y la estrategia política de la formación, comprobaremos si se produce o no una congruencia entre las posturas de los votantes y las del partido (Hernández-Carr, 2011b). Igualmente, la extensa literatura sobre la derecha radical populista nos servirá para contextualizar y contrastar la información extraída de las entrevistas. Finalmente, a partir de diferentes encuestas trataremos de comprobar si las posturas y opiniones de los votantes de PxC se enmarcan en corrientes de opinión más amplias entre la población catalana.

En cuanto a la estructura del texto, consta de tres apartados que recogen los tres ámbitos que el trabajo de campo ha señalado como centrales de cara a entender la atracción de los votantes hacia PxC. En el primer apartado analizaremos diferentes elementos de la trayectoria electoral y valores ideológicos de los votantes que muestran que el partido se está beneficiando de forma directa de procesos de largo recorrido como son el desalineamiento electoral y la desafección política. Asimismo, explicaremos cómo el partido ha conseguido posicionarse como una oferta política legítima para vehicular parte del voto de protesta contra los partidos políticos tradicionales. En el segundo apartado abordaremos la postura del partido y de los votantes respecto al debate territorial Catalunya-España (el "eje nacional”). La relevancia de esta temática radica en el hecho que, a nuestro entender, evitar ser vinculado al ultra-españolismo es el factor clave en el intento del partido de distinguirse de la estigmatizada extrema derecha española. En el tercer apartado del texto expondremos los que consideramos que son los principales motivos del fuerte rechazo a la inmigración expresado por los entrevistados y analizaremos por qué consideran que el voto a PxC es una buena respuesta frente a este fenómeno. Finalmente, en el cuarto apartado apuntaremos las principales conclusiones del texto y señalaremos algunos interrogantes que deberán ser abordados en futuros estudios.

\section{De la desalineación electoral al voto de protesta}

El desalineamiento electoral y la desafección política han sido señalados como un paso previo imprescindible para que una parte del electorado esté disponible y pueda ser "captado" por las formaciones de derecha radical populista (Rydgren, 2005). El desalineamiento electoral ha sido definido como "la pérdida de influencia de la identificación con un partido (y de otros valores políticos como la ideología izquierda-derecha) sobre el voto" (Anduiza y Bosch, 2004: 219). A este proceso de largo recorrido se le ha sumado una creciente desconfianza y criticismo de una parte de la población hacia las elites gobernantes (Taguieff, 2007). Un fenómeno que, no sin polémica, ha venido a conocerse como desafección política.

La consecuencia de estos dos procesos sería la existencia de un mayor número de electores "disponibles" y un aumento de la volatilidad electoral que puede favorecer la irrupción de nuevas formaciones. Asimismo, posibilita un mayor rédito electoral de los discursos de protesta contra los partidos tradicionales (Ignazi, 2003). 
En el caso de España y Catalunya, existen claros "síntomas” de que estos procesos se están intensificando en los últimos años. Por un lado, se ha producido un debilitamiento de la identificación partidista y un descenso en la valoración que los electores hacen de los partidos tradicionales y, por otro, se están manifestando con cada vez mayor intensidad determinados comportamientos electorales que responden a estos cambios en las actitudes políticas (aumento de la abstención, del voto en blanco y nulo, $y$, del voto a partidos minoritarios de protesta) (Vallès et al., 2008; Pont y Solís, 2009).

El trabajo de campo desarrollado muestra que la emergencia de PxC se enmarca dentro de este proceso y que el partido está obteniendo un rédito político y electoral del mismo. Tal y como desarrollaremos a continuación, sus electores no sólo presentan claros síntomas de distanciamiento y resentimiento hacia los partidos tradicionales, sino que se identifican con el discurso de PxC en relación a cuestiones como los valores políticos izquierda-derecha. Asimismo, ven al partido como un instrumento útil para protestar contra el sistema político.

\subsection{Un votante "libre" de ataduras}

Los votantes de PxC presentan un claro rechazo hacia dos valores políticos que tradicionalmente han servido para orientar las opciones electorales de los ciudadanos. Nos estamos refiriendo al eje izquierda-derecha y al vínculo entre clase social y orientación del voto (el "voto de clase"). En relación al eje izquierda-derecha, éste ha sido descrito como el principal instrumento para ubicar ideológicamente a los electores y a los partidos. No obstante, a lo largo de las últimas décadas se ha abierto un debate que apunta, entre otras cuestiones, la posibilidad de que se esté produciendo un debilitamiento de este eje y un descenso de su relevancia en el escenario político y electoral (Mair, 2007).

Los partidos de derecha radical populista se han caracterizado por el rechazo a situar su postura ideológica dentro del eje izquierda-derecha. En esta misma línea, Plataforma per Catalunya ha afirmado que su propuesta política rompe los esquemas tradicionales de izquierda y derecha y constituye una síntesis de las "mejores" propuestas de cada ámbito ideológico. El trabajo de campo ha mostrado que los votantes de PxC se sienten "cómodos" con esta propuesta política. Esto es así ya que afirman que no les importa excesivamente cuál es el posicionamiento exacto del partido en relación a este eje y que lo que perciben es que se trata de un proyecto político ajeno a estos debates "ideológicos". El partido se basaría, según los entrevistados, en propuestas concretas y pragmáticas que no tienen porqué ser circunscritas a un eje ideológico concreto.

Esta percepción de la relación entre PxC y el eje izquierda-derecha concuerda con su propia forma de aproximarse a dicho eje. La gran mayoría de entrevistados considera que en la actualidad este eje de división ideológica ha perdido relevancia y ya no tiene tanto sentido como en el pasado. Asimismo, apunta que no es especialmente significativo para ellos a la hora de orientarse ante la oferta política 
existente. En este sentido, una mayoría rechaza adscribirse de forma permanente en cualquiera de los dos lados de este eje, mientras que el resto, pese a que también señala la pérdida de relevancia de este eje, sigue ubicándose en alguno de los dos lados del mismo.

A la hora de argumentar por qué consideran que este eje ha perdido sentido encontramos una gran similitud discursiva entre los entrevistados. No se señala su falta de comprensión del significado de estos términos ni se niega completamente la existencia de diferentes posturas ideológicas dentro del debate político ${ }^{5}$, sino que se critica lo que se percibe como una división ideológica demasiado rígida que no satisface su forma de entender el juego político. Así, frente a lo que se ve como un excesivo encasillamiento ideológico se aboga por una postura "flexible" en que se tenga la "libertad" para escoger lo que más les interesa de cada uno de los polos en función de las circunstancias y de la evolución de sus opiniones e intereses. Este discurso encaja plenamente con la propuesta de PxC en el sentido de "adoptar" las mejores propuestas de cada uno de los polos del eje ideológico.

\section{Un voto de clase en declive}

El debilitamiento del "voto de clase", entendido como el vínculo electoral entre los miembros de una clase social y los partidos creados para representar a dicha clase social, ha contribuido fuertemente al proceso de desalineamiento y volatilidad electoral experimentado por las sociedades occidentales (Anduiza y Bosch, 2004). Dentro de este proceso ha sido especialmente significativo el debilitamiento del vínculo entre una parte de la clase trabajadora y el campo político de la izquierda (Michelat y Simon, 2004). El debilitamiento de este vínculo habría posibilitado que una parte de la clase trabajadora, especialmente las generaciones más jóvenes, pasase a engrosar la base electoral de las formaciones de derecha radical populista (Bjorklund y Andersen, 2007). Un proceso que habría sido clave en el auge electoral y en la obrerización del voto experimentada por la derecha radical populista a partir de la década de los noventa ${ }^{6}$ (Perrineau, 2005).

Las elecciones autonómicas catalanas del 2010, momento en que se realizaron las entrevistas, significaron la primera penetración significativa de PxC en la región metropolitana de Barcelona, especialmente en áreas de clase trabajadora marcadas

\footnotetext{
${ }^{5}$ Únicamente un pequeño grupo entre los entrevistados más jóvenes (entre 18 y 30 años) señala que no entiende la diferencia entre izquierda y derecha. Éstos se han abstenido en todas o prácticamente todas las elecciones y se han activado políticamente con la aparición de PxC.

${ }^{6}$ Las explicaciones sobre los motivos de este voto de clase trabajadora a la nueva extrema derecha son múltiples y polémicas. Para una descripción de las diferentes posturas véase los trabajos de Achteberger y Houtman (2006) y Bjorklund y Andersen (2007).
} 
por un claro predominio electoral del partido socialista (PSC-PSOE). ${ }^{7}$ Significativamente, muchos de los entrevistados se auto-ubican en la categoría "clase trabajadora” y señalan que en su entorno familiar hay una "tradición" de voto a partidos de izquierdas. En este sentido, el PSOE es el partido más mencionado como una opción de voto ejercida en algún momento de su pasada trayectoria electoral o practicada por gente de su entorno más inmediato. No obstante, los entrevistados, especialmente los jóvenes de clase trabajadora ${ }^{8}$, manifiestan claramente que en su caso este vínculo, que siguen percibiendo en sus padres, se ha roto. En algunos casos nunca siguieron la orientación de sus padres y en otros sí que lo hicieron por una especie de "inercia familiar". En cualquier caso, todos afirman de forma rotunda que la temática inmigratoria y la crisis económica han supuesto la ruptura definitiva de su posible vínculo con los partidos de izquierda.

Una parte significativa de los jóvenes de clase trabajadora entrevistados no sólo apuntan su escasa identificación con los partidos de izquierdas si no que manifiestan una fuerte adhesión a PxC. Muchos de ellos, sin estar afiliados al partido, afirman hacer una campaña activa de captación de votantes entre sus redes personales. Significativamente, muchos contraponen la receptividad de las personas de su misma edad con la dificultad de convencer a sus padres y a gente de edad avanzada para que voten al partido. Pese a tener una postura también fuertemente contraria a la inmigración, las generaciones de mayor edad se resisten a cambiar su voto, ya sea porque mantienen una fuerte adhesión a otro partido o porque relacionan a PxC con la extrema derecha franquista.

\subsection{El partido como instrumento para canalizar el voto de protesta}

El trabajo de campo ha mostrado de forma clara que los entrevistados se sienten ajenos y distantes del debate partidista y muy críticos con los partidos mayoritarios del sistema político español y catalán. Se crítica que las formas y el lenguaje del debate les resultan distantes cuando no ininteligibles y que las temáticas sobre las que gira el debate político no son, según ellos, las que realmente interesan a la gente "normal”. Todo ello les lleva a la conclusión de que han sido excluidos del sistema político y a expresar un fuerte resentimiento hacia todos los partidos tradicionales. No obstante, este sentimiento de alejamiento crítico del debate partidista no implica un desinterés por la política. De hecho, muchos exponen cómo en los últimos tiempos han sentido una creciente alarma por la situación del país y la suya propia,

\footnotetext{
${ }^{7}$ Nos estamos refiriendo a municipios como l'Hospitalet de Llobregat o Santa Coloma de Gramenet. Para un análisis de este proceso véase Hernández-Carr (2011a).

${ }^{8}$ Siguiendo la propuesta de Mayer (2003) consideramos jóvenes a los menores de 40 años.
} 
y una urgencia por encontrar formas de canalizar políticamente sus preocupaciones y su descontento.

La abstención electoral, una opción muy presente en la trayectoria electoral de los votantes ${ }^{9}$, lejos de presentarse como una muestra de conformidad con el estado de cosas se expone como la respuesta ante una oferta política que se considera indiferenciada y poco atractiva para sus intereses personales. Asimismo, un gran número de entrevistados señala su voluntad de apoyar a partidos pequeños que puedan regenerar el sistema político y abrir espacios para que se oigan "otras voces" desde dentro del sistema. Así, destaca que muchos han visto la emergencia del partido Ciutadans ${ }^{10}$ como algo especialmente positivo y señala haber votado o plantearse el voto tanto a este partido como a otras formaciones extraparlamentarias (especialmente a partidos ecologistas y animalistas). En este sentido, el apoyo a PxC es presentado también como una apuesta por dar un espacio dentro del sistema a nuevas voces y, al mismo tiempo, enviar un fuerte mensaje de protesta al establishment político.

La idea de que el voto a PxC sirve para enviar un mensaje de protesta al sistema político tradicional parte de la premisa de que el partido es un instrumento útil y legítimo para canalizar su resentimiento hacia las elites políticas. Las entrevistas han mostrado cómo esta imagen del partido se construye entre los votantes a partir de dos consideraciones. Por un lado la percepción de que es un partido "diferente” a los partidos tradicionales y, por otro, la creencia que su crecimiento es un mensaje de protesta radical contra el sistema político pero no constituye un peligro para el sistema democrático.

La imagen de PxC como un partido diferente y ajeno al establishment político se edifica, en nuestra opinión, a partir de dos factores. En primer lugar, el partido es considerado "valiente" por remar contracorriente y plantear cosas que, según su visión, sólo les van a llevar a problemas con los “poderosos”. El rechazo que suscitan sus propuestas y el propio partido entre los grandes partidos es visto como una señal de que el partido incomoda al sistema político tradicional y no pertenece al mismo. En este sentido, lejos de ver a PxC como un actor que se "aprovecha" del malestar de la ciudadanía, lo ven como un partido que "se la juega" por defender los intereses de los autóctonos. El segundo factor es que el partido utiliza un lenguaje y presenta unas propuestas que les resultan comprensibles y cercanas. En este sentido,

${ }^{9}$ La abstención es mencionada como una opción practicada o contemplada por muchos antes de haber conocido la oferta política de PxC. La atracción de votantes entre los abstencionistas es una de las principales conclusiones del estudio de Pardos-Prado y Molins (2010) sobre el voto a PxC.

${ }^{10}$ Este partido fue creado el año 2006 y obtuvo representación en el parlamento catalán en las elecciones autonómicas celebradas ese mismo año. 
se insiste en que, a diferencia de los partidos tradicionales, PxC desarrolla un lenguaje directo y claro con el que conectan fácilmente. ${ }^{11}$

En cuanto a la segunda variable, la gran mayoría de entrevistados considera que el partido no es una amenaza real para la democracia. En todos los casos se da por descontado que, actualmente, el sistema democrático está tan asentado en España que es imposible que un partido político como PxC pueda ponerlo en peligro. Algunos apuntan su convicción de que se trata de un proyecto político que en ningún caso contiene elementos contradictorios con el sistema democrático. Otros, en cambio, sí que ven ciertos peligros debido a lo que perciben como posturas antidemocráticas y excesivamente radicales de algunos miembros del partido. No obstante, afirman estar convencidos de que el partido nunca llegará a alcanzar el poder y que la obtención de unos pocos parlamentarios no entraña peligro alguno para el sistema democrático. Un razonamiento que les lleva a concluir que otorgarles su voto en estos momentos es un acto inofensivo.

La consideración de que el crecimiento de la nueva extrema derecha es un revés para los partidos tradicionales pero no pone en peligro el sistema democrático ha sido señalada como clave para que estos partidos reciban gran parte de sus votos (Hernández-Carr, 2001c). El recuerdo de los regímenes anti-democráticos instaurados en Europa en el periodo de entreguerras por movimientos de extrema derecha, junto al apoyo mayoritario entre la población al sistema de democracia representativa, hacen que muy difícilmente un partido considerado abiertamente antidemocrático pueda obtener un importante apoyo electoral. En este sentido, la clave del éxito de la nueva extrema derecha europea habría sido colocarse en una posición adecuada para explotar el resentimiento anti-establishment de la población sin ser vistos como un peligro para la democracia (Rydgren, 2007).

\section{Entre el ultra-españolismo y el nacionalismo catalán: en busca de la tercera vía}

La participación de Josep Anglada y de algunos de los militantes de la formación en partidos y movimientos ultra-españolistas ha hecho que el partido haya estado sometido a un escrutinio constante en relación a esta cuestión. La importancia de si el partido se inscribe dentro de este ámbito político o no se encuentra en el vínculo que, a ojos de la opinión pública, existe entre el ultra-españolismo radical y la extrema derecha anti-democrática. A nivel histórico, la experiencia de la dictadura franquista ha asentado en el imaginario colectivo, especialmente en un territorio

${ }^{11}$ Estos atributos, valentía y claridad, eran en muchos casos atribuidos directamente a Anglada más que al propio partido. No obstante, también se realizaban críticas a su figura. En particular, se señala lo que se considera que es una falta de preparación para la primera línea del debate político. 
como Catalunya, una conexión directa entre extrema derecha anti-democrática y nacionalismo ultra-españolista. Asimismo, los partidos y movimientos extraparlamentarios que actualmente se inscriben dentro de este ámbito político desarrollan una "defensa" de la unidad de España cuyo discurso enlaza con una exaltación, más o menos explícita, de la dictadura Franquista y se han visto involucrados en actos de violencia política y callejera (Rodríguez, 2010).

La trayectoria política de PxC muestra que, más allá de las preferencias individuales de sus miembros, el partido no ha hecho de la unidad de España un tema de agitación política. Igualmente, tampoco se ha enmarcado abiertamente dentro del ámbito del nacionalismo catalán. En este sentido, la apuesta de Plataforma puede entenderse como el intento de situarse en un punto intermedio y no conflictivo en relación al "eje nacional". El partido trataría de superar el eje de identificación nacional España-Catalunya del electorado catalán a partir del nuevo eje autóctonosextranjeros (Casals, 2006). Podría decirse que, al igual que las formaciones de derecha radical europeas rechazan situarse en relación al eje izquierda-derecha con el objetivo de aglutinar un apoyo electoral transversal, Plataforma pretende hacer lo mismo en torno al polémico eje nacional ${ }^{12}$.

Esta no ubicación dentro de un determinado campo político nacionalista es un elemento que diferencia claramente al partido tanto respecto a la extrema derecha española como a la mayoría de partidos de la nueva extrema derecha europea (Hernández-Carr, 2011a). A continuación explicaremos cómo el partido se ha visto obligado a ir concretando algunos elementos de su postura respecto al debate territorial y analizaremos cómo perciben los votantes dicha postura y si encaja o no con sus planteamientos al respecto.

\subsection{Buscando un perfil propio}

La postura principal del partido sigue siendo la de no incidir en el debate territorial y señalar que éste no es el debate que realmente interesa a la ciudadanía. No obstante, con el paso del tiempo el partido se ha visto obligado a ir concretando, o creando, su propio posicionamiento respecto a este debate. El crecimiento del partido más allá de la política municipal y su pretensión de acceder a la política parlamentaria habría acelerado la necesidad de desarrollar un cierto perfil propio que le permita tener presencia en el debate público y ofrecer algún tipo de respuesta a las inquietudes de su potencial electorado. En este sentido, pese a que el partido ha continuado

${ }^{12}$ Un ejemplo de ello lo encontramos en el hecho que, en relación al polémico debate del Estatut de Catalunya, la formación no adoptó una postura oficial (a favor o en contra) y dio libertad de voto a sus regidores municipales. 
ofreciendo importantes y desconcertantes giros respecto a su postura ${ }^{13}$, hay dos elementos que, especialmente a partir de su intento de acceder al parlamento catalán en el año 2010, han ido ganando peso en su discurso y conforman un incipiente posicionamiento respecto a esta temática.

En primer lugar, el partido ha incrementado sus mensajes afirmando explícitamente que rechaza la independencia de Catalunya. Las acusaciones recibidas, especialmente a lo largo del 2011 por parte de sectores del nacionalismo español acusando al partido de "hacerle el juego" al nacionalismo catalán o, directamente, de haber derivado en una formación soberanista han hecho que el partido emita comunicados oficiales en que se rechaza de forma rotunda que apoye la independencia de Catalunya ${ }^{14}$. En segundo lugar, ha desarrollado un discurso tratando de acentuar su "catalanidad", a partir de la repetición de ideas como que el partido se siente "orgulloso" de su pertenencia a Catalunya y que es un partido "muy catalán". En este sentido, ha desarrollado un discurso sobre su voluntad de "defender" a Catalunya en contraposición a lo que ha calificado como un "centralismo rancio". Una postura en la que destacan sus ataques al Partido Popular acusando a esta formación de promover el anti-catalanismo.

En definitiva, consideramos que la postura del partido puede ser entendida como el intento de construir una tercera vía que se sitúe a medio camino entre el nacionalismo español y el catalán. Esta postura puede sintetizarse en la idea de conjugar un rechazo a la independencia de Catalunya con una supuesta defensa de los intereses de los catalanes frente a lo que se señala como un ultra-españolismo excesivamente centralista y poco respetuoso con las "particularidades" catalanas. Esta postura trata de preservar su equidistancia o no posicionamiento explícito dentro del nacionalismo español o catalán y sigue siendo un discurso ambiguo que en ningún caso revela cuál es exactamente el modelo territorial que propone el partido. No obstante, sí que ofrece "pistas" a su electorado potencial sobre qué es lo que rechaza el partido.

\subsection{Una postura coincidente: ni independentismo ni anti-catalanismo}

El trabajo de campo ha mostrado que entre los votantes existe una notable coincidencia con las premisas centrales del posicionamiento del partido. La premisa principal y en la que coinciden prácticamente todos es que el debate territorial acapara demasiada atención y que, en estos momentos, este no es un tema priorita-

${ }^{13}$ Sobre estos giros en su postura véase el artículo de Xavier Casals " L'embolic identitari de Plataforma per Catalunya” publicado en el periódico Ara 27/09/11.

${ }^{14}$ Véase, por ejemplo, el comunicado del 23/09/11 en que se afirma que, ante los "falsos rumores” sobre su independentismo, “(...) queremos manifestar una vez más que Plataforma per Catalunya no ha apostado ni apostará en el futuro por la independencia de Catalunya”. 
rio para ellos y no debería serlo en el debate político general. Incluso entre aquéllos que se declaran independentistas, se concluye que esta temática debe ser aparcada temporalmente para hacer frente a lo que consideran que debe ser "resuelto" de forma urgente: la inmigración y la crisis económica. En este sentido, coinciden con la propuesta de PxC de dejar en un segundo plano este eje de división política y centrarse en la contraposición entre autóctonos y extranjeros.

La demanda de obviar el eje nacional, o no darle excesiva importancia, no debe ser confundida con una falta de postura al respecto. Los entrevistados sí que tienen una postura clara en relación a algunos aspectos del debate y sí que piden que haya una cierta coincidencia con la postura de PxC o, como mínimo, que no haya una excesiva contradicción. En este sentido, pese a que su voto al partido no se decide principalmente en función de su postura respecto a esta temática, sí que conocen los parámetros centrales del posicionamiento del partido y necesitan sentirse mínimamente "cómodos" con su postura.

Los elementos en que los entrevistados se posicionan de forma clara y en que piden, y creen obtener, afinidad por parte de PxC coinciden con los dos elementos que hemos apuntado anteriormente en relación a la postura del partido. En primer lugar, exceptuando a los escasos entrevistados que se declaran independentistas, hay una clara mayoría que se opone a la independencia de Catalunya. Dicha postura se justifica o bien apelando a la necesidad de aparcar este debate o bien directamente señalando su preferencia por la "unidad territorial” de España. El rechazo a la independencia se acompaña de la crítica a lo que se ve como una constante agitación de este tema por parte del nacionalismo catalán. Un nacionalismo que crea debates “artificiales” y desvía la atención de los “verdaderos” problemas. Respecto a esta cuestión, todos los entrevistados afirman tener la certeza de que PxC es un partido que no tiene como objetivo la independencia de Catalunya.

El segundo elemento atañe a lo que se considera que en ocasiones es un "trato injusto" hacia Cataluña desde España, especialmente en relación a la contribución económica al conjunto del estado ${ }^{15}$. Se considera que desde determinados ámbitos se instrumentalizan estos debates y que, en ocasiones, van en contra de los intereses de "los catalanes". En este sentido, gran parte de los entrevistados responsabiliza directamente al Partido Popular de esta situación y señala que, en ocasiones, dicho partido ha sido desleal hacia los catalanes al supeditarlos a sus intereses electorales en el resto de España. El PP es visto como un partido "obsesionado" con el debate territorial y que, al igual que el nacionalismo catalán, constantemente trata de extraer un rédito político del mismo. A consecuencia de esta postura, y de otros

${ }^{15}$ Muchos entrevistados señalan explícitamente que creen que hay que corregir el "déficit fiscal” de Catalunya y apoyan la creación de un nuevo modelo con el objetivo de que Catalunya disponga de más recursos económicos. 
factores como su supuesto elitismo, prácticamente todos los entrevistados desestiman la opción de votar al PP en unas elecciones autonómicas ${ }^{16}$.

Este rechazo al Partido Popular contrasta con la simpatía y el apoyo que un gran número de ellos expresa por el partido Ciutadans. La simpatía hacia este partido se basa tanto en que -como hemos señalado anteriormente- es visto como un partido ajeno al establishment político, como en una coincidencia con sus posturas en materia de lengua y debate territorial. Significativamente, les atrae lo que, a su juicio, es la apuesta del partido por dejar fuera del debate los temas identitarios y no malgastar energías y recursos en estos temas. Se ve a Ciutadans como un partido que defiende la unidad territorial de España pero que, a diferencia del PP, aboga por no agitar y polemizar con estos temas y dejarlos a la "libre elección" de cada persona.

El rechazo al Partido Popular y la simpatía por las posturas de Ciutadans es, a nuestro entender, un elemento altamente significativo respecto a la postura de los entrevistados. Denota lo que podría comprenderse como un autonomismo o catalanismo no identitario. Esto es, la voluntad de defender los intereses de los catalanes ante lo que se percibe como ataques injustos hacia Catalunya, pero sin hacerlo desde posiciones propias del nacionalismo catalán que puedan cuestionar la unidad territorial española. Asimismo, condensa la postura que consideran que tiene el partido y que resulta satisfactoria para una mayoría de éstos. La síntesis de la imagen que los votantes tienen de PxC es que es un partido que apuesta por la permanencia en España pero sin "perderse" en debates estériles y defendiendo "lo mejor" para Cataluña. La idea de la defensa de los intereses de los catalanes se acompaña de la creencia de que si bien PxC no es un partido nacionalista o independentista sí que es un partido "muy catalán”. Esta percepción se construye a partir de factores como que el partido ha nacido y crecido en zonas consideradas "muy catalanas" o "muy nacionalistas" (especialmente por ser Vic el punto de referencia del partido) o que el catalán es el idioma usado habitualmente tanto por los principales dirigentes como por la propaganda de la formación.

Este factor, el "pedigrí catalán" del partido y su supuesta defensa de los intereses de los catalanes, es especialmente importante de cara a que el partido supere el estigma de la vinculación a la extrema derecha españolista. Una clara muestra de ello es que los entrevistados que afirman sentirse próximos al nacionalismo catalán y/o que recelan especialmente del vínculo entre PxC y la extrema derecha españolista, señalan que, pese a que "saben" que "en el fondo" Anglada y otros miembros del partido son ultra-españolistas, el hecho de que no hagan bandera de esta temáti-

${ }^{16}$ Es significativo que esta argumentación la desarrollan incluso aquellos que sí que contemplan al PP como una opción electoral plausible y afirman que seguramente le darían su voto en unas elecciones generales. En este sentido, todos los entrevistados coinciden en señalar que en unas elecciones autonómicas es más "útil” votar a PxC que al PP. 
ca y que el partido les transmita una "sensación" de catalanidad permite que obvien este factor y le den su voto.

\section{Inmigración: el trampolín hacia la nueva extrema derecha}

El rechazo a la inmigración es, sin duda, el gran reclamo electoral de Plataforma per Catalunya. Antes de conocer la existencia de PxC, los entrevistados ya tenían en la inmigración su principal preocupación y buscaban una oferta política que diese salida a sus posturas. En este sentido, el partido está captando una demanda electoral que ya existía y que buscaba un referente político que reflejase sus posturas (Casals, 2010). Siguiendo la propuesta de Evans e Ivaldi (2002), puede decirse que los entrevistados presentan una "visión etnocéntrica" de los problemas sociales que les lleva a relacionar esta temática con multitud de fenómenos. La forma en que articulan su discurso muestra que la inmigración es un catalizador a través del cual expresan un amplio abanico de preocupaciones.

En el presente apartado desarrollaremos dos elementos que, a nuestro entender, exponen de forma complementaria el papel del rechazo a la inmigración en el voto a PxC. En primer lugar detallaremos las que consideramos que son las tres temáticas alrededor de las cuales se articula su rechazo a la presencia de población extranjera. En segundo lugar, mostraremos cómo el fenómeno migratorio es la base a partir de la cual los entrevistados desarrollan una cierta lectura de la situación social y política del país que les lleva a la conclusión de que en estos momentos es necesaria una "respuesta contundente" como la que ofrece PxC.

\subsection{Los tres vectores principales del rechazo a la inmigración}

Las tres temáticas que concentran el rechazo de los votantes hacia la población extranjera son: las preocupaciones materiales (trabajo y ayudas públicas), un sentimiento de inseguridad vinculado tanto a la delincuencia como a un entorno físico que cambia a gran velocidad y el rechazo a la inmigración musulmana. Dado que son tres temáticas de gran envergadura y que pueden ser abordadas desde múltiples ángulos, nos limitaremos a subrayar el núcleo del discurso de los entrevistados y a apuntar qué vinculo puede establecerse entre su postura y su decisión de apoyar a PxC.

\subsubsection{Proteger a los de casa}

La necesidad de proporcionar una mayor “protección” material a la población autóctona es, seguramente, la demanda más repetida y claramente expuesta a lo largo de las entrevistas. Dicha protección se refiere principalmente al acceso al mercado laboral y a los recursos públicos. A la hora de concretar qué se entiende por dar una mayor protección en estos ámbitos se produce una significativa contra- 
dicción en el discurso. Así, inicialmente se señala que, dado que supuestamente existe un trato de favoritismo por parte de las administraciones públicas hacia la población extranjera, lo que piden es un trato en igualdad respecto a ésta. No obstante, al ser preguntados por el principal lema del partido ("Primero los de casa"), señalan estar totalmente de acuerdo con el mismo y desarrollan un discurso en que se defiende la necesidad de dar prioridad a la población autóctona en el acceso al empleo y a los recursos públicos.

El lema de PxC y la postura de los entrevistados conectan claramente con las propuestas de "preferencia nacional" desarrolladas por la derecha radical populista europea. Estas propuestas se han dirigido principalmente a un electorado alarmado por la precarización de sus condiciones de vida materiales y habría servido para captar las demandas de protección social de una parte de la clase trabajadora ${ }^{17}$ (Perrineau, 2005). De cara a comprender este proceso, las tesis explicativas del interés económico (Eatwell, 2003) y de la competición étnica (Rydgren, 2007) apuntan que son principalmente aquellos que están en una situación de precariedad económica y de competencia directa con la población extranjera los que pasarán a apoyar a este tipo de partidos. La competición por unos recursos escasos llevaría a aquellos que no han podido acceder a dichos recursos, o que temen perder el acceso, a adoptar una postura de exclusión hacia el grupo social minoritario. En este sentido, una parte de los entrevistados señalan explícitamente que, a su juicio, existe una competición directa entre ellos y la población extranjera. Así, se considera que el supuesto acaparamiento de los recursos (laborales y asistenciales) por parte de la población extranjera es la causa de sus dificultades económicas.

Pese a la relevancia de este discurso, creemos que entender esta demanda de una mayor protección como un anhelo que afecta únicamente a aquellos que compiten de forma directa por unos recursos escasos no proporciona una comprensión completa del fenómeno. La necesidad de adoptar una perspectiva más amplia ha sido defendida por diferentes especialistas en el fenómeno de la nueva extrema derecha. Así, Minkenberg (2000) señala que son todos aquéllos que "temen perder algo", y no sólo los que están en una situación de clara privación económica, los que se pueden ver atraídos por los discursos de protección social de la nueva extrema derecha. Asimismo, la pérdida de la posición socio-económica (el estatus social), o el miedo a la pérdida, puede ser tan o más importante para la comprensión de este fenómeno que unas condiciones objetivas de privación económica (Rydgren, 2007).

$\mathrm{Y}$, efectivamente, en el relato de un gran número de entrevistados se enfatiza su temor a lo que se ve como un retroceso, o un inminente retroceso, de su posición social. Señalan que hace unos pocos años no hubiesen imaginado la situación de precariedad económica en la que se encuentran y, al mismo tiempo, expresan su

${ }^{17}$ Las encuestas apuntan que en Catalunya se ha producido un incremento de las posturas que abogan por priorizar a la población autóctona/nacional en el acceso a determinados bienes y servicios (especialmente en el acceso a las ayudas públicas) (Méndez, 2009). 
angustia por lo que temen que pueda ser un futuro mucho peor que el presente. Este miedo al futuro, a una situación de exclusión social, les lleva a mostrar una fuerte demanda por una acción contundente que garantice su protección como miembros de la comunidad "nacional". En este sentido, puede decirse que el apoyo a PxC se encuentra espoleado por el miedo a quedar atrapados en una situación que no se ajusta a sus expectativas, sean cuales sean, y es una apelación a frenar lo que se ve como un proceso de movilidad social descendiente.

\subsubsection{De la inseguridad a la nostalgia populista}

La creencia de que existe un vínculo entre inmigración e incremento de la delincuencia, y la demanda de posturas mucho más punitivas al respecto es unánime entre los entrevistados. El trabajo de campo ha mostrado que esta temática es claramente una preocupación de primer orden para los votantes de PxC. Esta postura conecta plenamente con el electorado europeo de la nueva extrema derecha para el que la conexión entre inseguridad ciudadana e inmigración se ha mostrado como uno de los principales motores del voto a estos partidos (Ivaldi, 2003).

Junto a la indiscutible centralidad de la delincuencia en sentido estricto entre las preocupaciones de los votantes, en las entrevistas se han apuntado también otros fenómenos que conviene tener en cuenta. En primer lugar, el relato de los entrevistados muestra que su sentimiento de inseguridad y rechazo a la inmigración no se basa únicamente en elementos vinculados a la delincuencia (robos, agresiones, etc.), sino que se nutre también de un amplio abanico de fenómenos que pueden entrar o no en el terreno de la ilegalidad. Así, se expone su incomodidad ante lo que se percibe como un cambio acelerado de su entorno urbano, tanto por la "excesiva" presencia de población extranjera en el espacio público como por los cambios en el tejido comercial. Asimismo, se da una gran relevancia a elementos relacionados con el "comportamiento inadecuado" de la población extranjera en el espacio público.

El relato de los entrevistados nos conduce a la propuesta explicativa desarrollada por Perrineau (1997) para explicar la relevancia de estos fenómenos en el voto al Frente Nacional francés. De acuerdo con este autor, los elementos que acabamos de describir generan una difusa sensación de malestar e inseguridad entre la población. Este malestar difuso, que define como inquietudes urbanas, se vería "confirmado" por los grandes episodios de delincuencia o de revueltas urbanas sucedidos en cualquier punto del territorio. La potencia de esta temática es que puede afectar tanto a aquellos que viven en barrios con altos porcentajes de inmigración como a los que no. La conclusión del autor es que la combinación de la delincuencia en sentido estricto con estas inquietudes urbanas es un ámbito del que la nueva extrema derecha puede extraer un gran rédito electoral.

Todos estos elementos conectan también con un discurso desarrollado por los entrevistados que, a nuestro entender, puede ser definido como un populismo nostálgico (Betz y Johnson, 2004). Dicho discurso, que se vincula especialmente al ámbito local (ya sea a nivel de barrio o de municipio), contrapone los fenómenos que acabamos de señalar (delincuencia, usos del espacio público inadecuados, etc.) 
con una visión nostálgica y elogiosa del pasado. Bajo esta mirada al pasado se enfatiza una supuesta falta de divisiones sociales y conflictos entre los vecinos. En esta línea, se subraya que, a diferencia del proceso actual, la emigración a Catalunya de trabajadores de otras regiones de España apenas fue conflictiva. Asimismo, se apunta que la llegada de inmigración extranjera habría llevado a la pérdida de los vínculos de solidaridad y reconocimiento mutuo existentes entre los vecinos, $\mathrm{y}$, al debilitamiento de su "identidad" colectiva.

El propio partido ha desarrollado y alimentado este discurso nostálgico. Así, especialmente en el ámbito de la política local se ha jugado con la idea de que PxC es un partido que lucha por preservar la sociabilidad e identidad de unos barrios y municipios que habrían crecido excesivamente. Asimismo, el partido remarca la idea de que hubo una gran convivencia entre catalanes de origen y emigrantes, y que la mejora de las condiciones de vida y del entorno urbano de muchos barrios y municipios se consiguió gracias a la unión y el esfuerzo de todos. Este discurso sobre un tiempo y un espacio pretérito, que se señala como más sencillo y abarcable, es una constante entre los partidos de derecha radical populista. Esta explotación de la nostalgia de una "época dorada" puede ser efectiva entre aquellos que perciben que su “mundo", físico y simbólico, está en proceso de cambio acelerado (Rydgren, 2007). En este sentido, este populismo nostálgico es un factor fundamental a la hora de comprender el vínculo que los votantes establecen con Plataforma per Catalunya.

\subsubsection{Islam: una amenaza a "nuestra forma de vida"}

El rechazo al Islam es una postura compartida por la gran mayoría de entrevistados. Asimismo, los inmigrantes de religión musulmana son señalados como el colectivo que más "preocupación” y rechazo genera. Estas posturas enlazan con el gran protagonismo que ha adquirido la "cuestión musulmana" en la sociedades occidentales. Así, desde finales de los años noventa esta temática ha entrado en el primer plano del debate político y se ha convertido en uno de los pilares del discurso de todos los partidos de nueva extrema derecha (Zuquete, 2008). Igualmente, Plataforma per Catalunya ha hecho del rechazo al Islam uno de sus principales instrumentos de movilización política (Casals, 2006).

La caracterización del Islam que desarrollan los entrevistados puede resumirse en dos ideas fuerza. La primera apunta que se trata de una religión retrógrada que implica una serie de prácticas y creencias que, en el caso de Catalunya, ya habrían sido superadas gracias al progreso de las sociedades occidentales. La segunda señala que se trata de una religión que tiene una clara voluntad de expansión e imposición de sus propias creencias. En este sentido, el Islam es señalado como un fenómeno que va más allá de la esfera religiosa y afecta a todos los ámbitos de la vida en sociedad. Consecuentemente, aquellos que no practican esta religión también van a sufrir sus prácticas retrógradas en cualquier ámbito de su vida privada.

A partir de estas premisas se desarrollan dos líneas argumentativas sobre qué es lo que la llegada del Islam está poniendo en peligro y debe ser defendido. Por un lado se destaca la amenaza que supondría para las conquistas sociales logradas por 
las sociedades occidentales (sistema democrático, igualdad de género, libertad de expresión, etc.). Dentro de este discurso se señala también que los creyentes musulmanes no respetan la separación entre la esfera pública y la religiosa y quieren que sus posturas sean acatadas por el conjunto de la población. No obstante, la segunda línea argumentativa se basa precisamente en la idea de que hay que defender la preeminencia en España de las tradiciones y del papel público del catolicismo.

Este discurso sobre la necesidad de defender la tradición católica del país es, a nuestro entender, un elemento especialmente significativo, tanto por el hecho de que es el argumento más empleado por los entrevistados como porque podría darnos pistas sobre qué tipo de elector se siente especialmente atraído por el discurso de oposición al Islam. Analizando este tipo de discursos, Storm (2011) ha señalado que el poder de movilización que la oposición al Islam ha adquirido en las sociedades europeas no se basa en parámetros estrictamente religiosos. De acuerdo con esta autora, lo que moviliza a una parte del electorado es la defensa de una identidad católica vinculada a una tradición nacional y a una idea de comunidad que va más allá del ámbito religioso. Los individuos que cuando se identifican como católicos lo hacen en relación a una tradición cultural-nacional o una herencia étnica, y no a una práctica religiosa, son los que presentan mayores actitudes anti-inmigración y los que se oponen de forma radical al inmigrante musulmán.

La hipótesis explicativa de Storm encaja con algunos elementos apuntados por los entrevistados. Así, la gran mayoría de éstos no se define como católico practicante sino como católico no practicante o incluso no creyente. Asimismo, al explicar su defensa de las "tradiciones" católicas especifica que no le mueve una creencia religiosa sino la idea de que las tradiciones católicas forman parte de las costumbres del país y deben mantenerse porque "siempre ha sido así". En este sentido, consideramos que, en el caso de los votantes de PxC, estamos ante una apelación a una identidad católica cultural y no religiosa. Siguiendo nuevamente la propuesta de Storm (2011), entendemos que esta apelación a las tradiciones "propias" del catolicismo es un instrumento para marcar y articular una identidad colectiva entre la población autóctona que les permita distinguirse del colectivo objeto de su rechazo (el inmigrante musulmán). Esta necesidad de dotarse de unos símbolos propios se nutre de la percepción de que, frente a una comunidad musulmana supuestamente homogénea y unida, la comunidad "nacional” ha perdido su sentimiento de solidaridad e identidad colectiva. Una situación que sería vista como una muestra de debilidad e indefensión y que se intentaría revertir mediante la apelación a unas tradiciones y una identidad católica comunes.

\subsection{Una situación fuera de control, un voto para restablecer el orden}

Los diferentes elementos que hemos ido apuntando (falta de protección material a los autóctonos, inseguridad, penetración del Islam) son la base a partir de la cual los entrevistados articulan un discurso global sobre la situación de la sociedad española y catalana y sobre la necesidad de apoyar una propuesta política como la de PxC. 
Este discurso gira alrededor de la creencia de que actualmente las sociedades española y catalana se encuentran inmersas en una situación de emergencia y de crisis sin precedentes.

El punto de partida y el eje de su relato es la idea de que la llegada de población extranjera constituye un proceso descontrolado que amenaza múltiples ámbitos de la vida social. Asimismo, pese a que esta percepción es previa y autónoma respecto a la crisis económica en la que se encuentra inmersa España, dicha crisis intensifica y, al mismo tiempo, justifica esta percepción. Finalmente, se apunta que la raíz del problema se encuentra en que los partidos políticos tradicionales han permitido, o han impulsado, el proceso migratorio. Estos partidos no sólo han "apostado" por la inmigración, sino que buscan sus votos y, por tanto, van a servir sus intereses. Este discurso concuerda con la idea de Taguieff (2007) de que los partidos de nueva extrema derecha y su electorado consideran que los partidos tradicionales habrían dejado totalmente desamparados a los autóctonos, especialmente a aquellos con menos recursos, y son considerados como "el partido del extranjero".

Esta lectura de la evolución social del país en los últimos años y de su estado actual se completa con una visión marcadamente pesimista respecto a su propio futuro y el del conjunto de la sociedad. Este miedo al futuro está muy presente en los relatos de los entrevistados y enlaza con los trabajos sobre la derecha radical populista europea que han enfatizado la importancia del "miedo al porvenir" y del sentimiento de inseguridad y amenaza como atributos esenciales para comprender las posturas de los votantes de estos partidos (d'Apollonia, 2007).

La conclusión a la que se llega ante este escenario es que urge actuar de forma contundente para frenar la evolución negativa del conjunto social y de su situación personal. En este sentido, hay una clara exhortación a la necesidad de adoptar medidas drásticas. Una muestra de esta demanda la encontramos en el hecho de que muchos entrevistados señalan explícitamente que, pese a no apoyar una vuelta a un régimen dictatorial como el franquista, sí que entendían que en la situación actual era necesario recuperar algunas de las medidas desarrolladas durante esa época con el objetivo de mantener el "orden social".

El apoyo a PxC es presentado como uno de los recursos disponibles para que se implementen medidas drásticas que permitan cambiar el rumbo que ha adoptado el conjunto de la sociedad. El apoyo al partido puede ser entendido como el recurso a una respuesta autoritaria ante un proceso que se juzga "fuera de control". Este anhelo por una respuesta autoritaria enlaza con los trabajos de la literatura sobre la derecha radical populista que han señalado tanto la importancia de las actitudes autoritarias entre los votantes (Mayer, 1997) como la existencia de "una concepción autoritaria del orden social” entre los partidos adscritos a esta familia de partidos (Rodríguez, 2006).

No obstante, para que un partido pueda beneficiarse de este anhelo es indispensable que los electores lo conciban como un instrumento útil para desarrollar esta "respuesta autoritaria". En el caso de PxC, las entrevistas han mostrado que esta creencia se fundamenta en su imagen de partido radical y valiente, $y$ en la idea que ha demostrado ser un partido eficaz a la hora de conseguir ciertos objetivos. Res- 
pecto al primer elemento, el apoyo a $\mathrm{PxC}$ es presentado como una respuesta extrema ante una situación extrema. En este sentido, la imagen del partido y de Anglada como una propuesta política radical parece jugar, hasta cierto punto, en su favor. Para que esto sea posible es importante que, como hemos explicado anteriormente, las propuestas del partido sean vistas como radicales pero no como un peligro real para la democracia. Esta imagen de radicalidad se conjuga con la idea de que el partido, y especialmente su líder Josep Anglada, no van a modificar su discurso y sus propuestas por el hecho de ser mal vistas por el resto de partidos políticos. La "valentía" del partido sería la garantía de que van a seguir ofreciendo respuestas contundentes ante una situación que así lo exige.

Las entrevistas han mostrado que para los votantes es importante la consideración de PxC como un partido que consigue resultados tangibles. Esta creencia se construye tanto a través de lo que se ve como un trabajo eficaz desarrollado en el ámbito de la política municipal (especialmente en municipios como Vic o El Vendrell), como de su capacidad para influir en las posturas de los grandes partidos políticos respecto a la inmigración. En este sentido, la polémica decisión del ayuntamiento de Vic en el año 2010 de no empadronar a población extranjera en situación irregular, y que PxC presentó como el resultado de su presión política y electoral (Hernández-Carr, 2011a), es señalada como la prueba de que el crecimiento del partido tiene consecuencia prácticas y de envergadura. Este episodio, muy presente en los relatos de los entrevistados, sería la muestra de que la implantación y la presión del partido en una zona concreta pueden acabar teniendo efectos sobre el conjunto del debate político de Catalunya y de España.

\section{Conclusiones y futuros interrogantes}

A lo largo del texto hemos ido detallando los principales factores explicativos del proceso de atracción de los electores hacia Plataforma per Catalunya. No hay duda de que la inmigración es el principal reclamo electoral del partido y el gran motor del apoyo expresado por los votantes. No obstante, el trabajo de campo ha señalado que otros factores (la desafección política, la desvinculación de PxC respecto a la extrema derecha españolista, etc.) son necesarios para que los electores, especialmente aquellos que no se adscriben al ámbito político de la extrema derecha, estén disponibles y se sientan suficientemente "cómodos" como para dar su voto al partido. Estas variables no directamente relacionadas con la inmigración aportan una información de gran interés de cara a comprender por qué algunos electores dan el salto a la nueva extrema derecha mientras que otros, también críticos con la inmigración, no lo hacen.

El texto ha apuntado algunos elementos sobre el perfil de los votantes que, a nuestro entender, son de gran interés y cuyo análisis deberá profundizarse en futuros estudios. Tal y como hemos señalado anteriormente, las elecciones autonómicas del 2010 supusieron la primera penetración del partido en zonas de la región metropolitana de Barcelona de clase trabajadora y con un tradicional predominio del voto 
de izquierdas. El trabajo de campo apunta la posibilidad de que dentro de este proceso el partido esté atrayendo principalmente a las generaciones jóvenes-adultas (menores de 40 años) que, pese a enmarcarse en ámbitos familiares y residenciales de clase trabajadora y con una tradición de voto izquierdista, no se sienten interpeladas por los parámetros de adscripción ideológica "clásicos” (eje izquierda-derecha, voto de clase). A partir de una falta de adscripción ideológica y de identificación partidista fuerte, estos electores se sienten atraídos por una nueva oferta política que consideran que da una respuesta a sus necesidades y preocupaciones más inmediatas.

Este perfil de población encaja con los resultados de Pardos-Prado y Molins (2010) sobre el voto a PxC en las elecciones municipales 2003 y 2007. Los autores concluyen que el electorado del partido está compuesto principalmente por antiguos votantes socialistas y abstencionistas que se han activado debido a la irrupción del partido. Asimismo, nos remite al proceso de atracción del electorado de clase trabajadora que, especialmente a partir de la década de los noventa, habría pasado a engrosar la base electoral de la nueva extrema derecha europea. Según Mayer (2003), los votantes de clase trabajadora menores de 40 años, ninistas (que no se ubican claramente a la izquierda o a la derecha de este eje) y con tendencia a caer en la abstención, han sido la base del apoyo electoral de partidos como el Frente Nacional francés. Se trataría de unos electores que al no presentar una gran politización respecto a temáticas vinculadas al eje izquierda-derecha tradicional (en relación a cuestiones como la política económica, el papel del estado, etc.) ni una identificación sólida con un determinado partido o ámbito político, son más susceptibles de sentirse atraídos por el discurso de la nueva extrema derecha (Ivarsflaten, 2005).

Respecto a esta incipiente penetración entre el electorado de clase trabajadora, es significativo que a raíz de los resultados de las elecciones autonómicas del 2010 el partido adoptase oficialmente una estrategia de crecimiento basada en "un giro social para acercar a PxC a la izquierda sociológica" ${ }^{18}$. Asimismo, las elecciones municipales y legislativas del 2011 confirmaron que el principal granero de votos del partido se había desplazado desde las comarcas del interior de Catalunya (Osona) a municipios de la región metropolitana de Barcelona como L'Hospitalet de Llobregat, Santa Coloma de Gramenet o Sant Boi de Llobregat. En este sentido, el futuro electoral del partido dependerá en gran medida de su capacidad para consolidar e incrementar su base electoral entre los electores de clase trabajadora emplazados en estos municipios.

Un elemento significativo de nuestro trabajo es que recoge el momento en que una parte del electorado catalán decide dar el "salto" a la nueva extrema derecha. No en vano las elecciones autonómicas del 2010 supusieron un gran incremento en

${ }^{18}$ La ponencia “Manifest pel Gir Social de PxC” fue aprobada en el Quinto Congreso del partido celebrado el 27 de marzo del 2011 en la ciudad de Vic. 
los votos de PxC respecto a citas electorales previas. Asimismo, prácticamente todos los entrevistados afirman que es la primera vez que van a dar su voto al partido. En este sentido, es interesante comprobar qué vínculo establecen los votantes con el partido. Si presentan su voto como un apoyo meramente coyuntural e instrumental o si existe una identificación que apunta a una mayor fidelidad electoral y a una relación con el partido que pueda ir más allá del voto (activismo, afiliación, etc.).

Respecto a esta cuestión, hemos encontrado dos posturas entre los votantes. Una parte de los entrevistados, principalmente los jóvenes de clase trabajadora, expresa una fuerte identificación con el proyecto de PxC y afirma estar dispuesto a desarrollar una implicación que vaya más allá del voto. Así, algunos de ellos contemplan la posibilidad de "ayudar" de alguna manera en la campaña electoral del partido y se plantean la opción de afiliarse al mismo. Dentro de esta voluntad de implicarse más allá del mero apoyo electoral destaca el constante activismo que, según estos votantes, despliegan y seguirán desplegando entre sus redes personales. Así, muchos entrevistados relatan cómo aprovechan múltiples ámbitos de relación social (familiar, escolar, laboral) para dar a conocer al partido y para desmontar la imagen de excesiva radicalidad que según ellos manejaban muchos de sus interlocutores. Este activismo y proselitismo entre las redes personales, especialmente por parte de gente que no se adscribe al ámbito de la extrema derecha tradicional, puede ser un arma muy potente para el crecimiento de una formación de estas características (Lazar, 1993).

El segundo grupo de entrevistados desarrolla un discurso basado en la idea de que PxC es un instrumento temporal para vehicular un mensaje de protesta contra los partidos mayoritarios y contra su postura en materia de inmigración. Estos votantes señalan que, pese a que han superado sus reticencias iniciales y les van a conceder su voto, no se identifican con el partido hasta el punto de asegurar su voto en futuras citas electorales ni de hacer apología pública a favor del mismo. Esta postura se expone a través de dos argumentos. El primer argumento apunta a las reticencias que aún despierta la relación entre PxC y la extrema derecha tradicional o anti-democrática. En este sentido, se apunta al pasado político de Anglada y, sobre todo, a lo que se califica como las actuales "malas compañías" del partido. Esto es, ciertos discursos de miembros del partido, o de gente próxima a él, que se consideran demasiado extremistas y se asocian con la extrema derecha tradicional y/o con una incitación a la violencia hacia la población extranjera. El segundo argumento remite a la idea de que no quieren establecer un vínculo permanente con ninguna formación política (incluida PxC) y, por tanto, no pueden predecir sus futuras decisiones electorales. Esta argumentación encaja plenamente con la apuesta de los entrevistados por la "flexibilidad" ideológica y política y su idea de ir escogiendo la mejor opción electoral según las circunstancias de cada momento.

Los dos argumentos que acabamos de exponer exponen algunos interrogantes clave de cara a la evolución futura del apoyo electoral al partido. La idea que hay que primar la flexibilidad ideológica y política y escoger la opción electoral en función de los intereses de cada momento es un argumento compartido no sólo por 
aquellos con una visión instrumental de su relación con PxC sino por la gran mayoría de entrevistados. El rechazo a una vinculación permanente con cualquier partido político es una postura fuertemente enfatizada por prácticamente todos los votantes. De tal manera que, siendo el rechazo a establecer una identificación partidista fuerte una de las principales características de sus votantes, resulta plausible apuntar la posibilidad de que el partido vaya a tener ciertas dificultades a la hora de consolidar una amplia base electoral estable y fiel a sus siglas. En este sentido, cabe destacar que el análisis de Pardos-Prado y Molins (2010) ya apuntaba que el partido tenía mayor capacidad para atraer a nuevos votantes que para retener a sus antiguos electores.

Las reticencias que genera la "proximidad" del partido, o de ciertos miembros del mismo, con la extrema derecha tradicional muestran que, pese a que los entrevistados no ven a PxC como una amenaza para el sistema democrático, éste sigue siendo un factor clave en la imagen que gran parte del electorado tiene del partido. En esta misma línea, muchos entrevistados han expuesto que personas de su entorno fuertemente contrarias a la población extranjera, principalmente gente de edad avanzada pero no sólo, no se deciden a votar al partido porque lo siguen relacionando con la extrema derecha franquista y ultra-españolista. De tal manera que parece acertado concluir que, en el caso de PxC, el estigma que pesa sobre la extrema derecha tradicional no ha sido superado del todo y sigue limitando su capacidad para convertir en votos a todo su electorado potencial. 


\section{Bibliografía}

Achterberger, P. y Houtman, D. (2006) "Why do so many people vote "unnaturally"?” A cultural explanation for voting behaviour', European Journal of Political Research, 45: 75-92

Anduiza, E. y Bosch, A. (2004) Comportamiento político y electoral, Barcelona, Ariel

Bartlett, J.; Birdwell, J.; Littler, M. (2011) "The new face of digital populism", Londres, DEMOS

Betz, H. G. y Johnson, C. (2004) "Against the current-stemming the tide: the nostalgic ideology of the contemporary radical populist right”, Journal of Political Ideologies, 9 (3): 311-27

Bjorklund, T. y Andersen, J. (2007) "La derecha radical populista en Escandinavia”, en: Miguel Ángel Simón, La extrema derecha en Europa desde 1945 a nuestros días, Madrid: Tecnos.

Casals, X. (2006) Ultracatalunya. Barcelona: L'esfera dels llibres.

--- (2009): La Plataforma per Catalunya: la eclosión de un nacional-populismo catalán (2003-2009), Working Papers n²74, Institut de Ciències Polítiques i Socials, Barcelona

d'Apollonia, A. (2007) "Xenofobia y extremas derechas en Europa”, en: Miguel

Ángel Simón, La extrema derecha en Europa desde 1945 a nuestros días, Madrid:

Tecnos.

Eatwell, R. (2003) “Ten theories of the extreme right”, en: Peter Merkl y Leonard Weinberg, Right-wing extremism in the twenty-first century, London: Frank Cass.

Erra, M. y Serra, J. (2008) Tota la veritat sobre Plataforma per Catalunya, Badalona, Ara Llibres,

Evans, J. (2005), 'The dynamics of social change in radical right-wing populist party support ', Comparative European Politics, 3, 76-101.

Evans, J. y Ivaldi, G. (2002) "Les dynamiques électorales de l'extrême droite européenne”, Revue Politique et Parlementaire, 1019: 67-68

Faniel, J. (2000) "Vote brun en banlieue rouge: une approache qualitative du vote populaire d'extrême droite à Seraing”, Les Cahiers du Cevipol, $\mathrm{n}^{\circ} 3$.

Hernández-Carr, A. (2011a) El largo ciclo electoral de Plataforma per Catalunya: del ámbito local a la implantación nacional (2003-2011), Working Papers n³00, Barcelona, Institut de Ciències Polítiques i Socials

--- (2011b) ¿La hora del populismo? Elementos para comprender el éxito electoral de Plataforma per Catalunya, Revista de Estudios Políticos, 153: 47-74

--- (2011c) La derecha radical populista en Europa: discurso, electorado y explicaciones, Revista Española de Investigaciones Sociológicas, 136: 141-160

Ignazi, P. (2003) Extreme right parties in western Europe. Oxford: Oxford University Press.

Ivaldi, G. (2003) “Enjeux sécuritaires et droites populistes en Europe”, en: Armand Colin, En quête de sécurité : Causes de la délinquance et nouvelles réponses. 
Ivarsflaten, E. (2005) "The vulnerable populist right parties: no economic realignment fuelling their electoral success", European Journal of Political Research, 44: 465-92.

Kitschelt, H. (1995) The radical right in Western Europe. A comparative analysis. Ann Arbor: University of Michigan Press.

Lazar, M. (1993) “Italie: pour comprendre la Ligue”, Revue Française de Science

Politique, 43 (6) : 1022-7.

Lipset, S. Y Raab, E. (1970) The Politics of Unreason: Right Wing Extremism in America, 1790-1970. New York, Harper \& Row.

Mair, P. (2007) "Left-Right orientations", en: Dalton y Klingeman (eds.) The Oxford Handbook of Political Behaviour, Oxford: Oxford University Press

Mayer, N. (1997) “Le vote Front National au pluriel”, Le Banquet, 10.

--- (2003) "Que reste-t-il du vote de classe? Le cas français”, Lien social et Politiques, 49: 101-11.

Méndez, M. (2009) “L’opinió dels catalans sobre la immigració”, Informes Breus, Barcelona, Fundació Jaume Bofill.

Michelat, G. y Simon, M. (2004) Les ouvriers et la politique. Permanence, ruptures, réalignements, Paris: Presses de Sciences Po.

Minkenberg, M. (2000), "The renewal of the radical right: between modernity and antimodernity”, Government and Opposition, vol. 35 n²: 170-188

Mudde, C. (2007) Populist radical right parties in Europe Cambridge: Cambridge University Press.

Norris, P. (2005) Derecha radical populista. Votantes y partidos políticos en el mercado Electoral, Madrid, Akal.

Pardos-Prado, S. y Molins, J. (2010) "The emergence of right-wing radicalism at the local level in Spain: the Catalan case”, International Journal of Iberian Studies, vol. 23, $\mathrm{n}^{\circ} 3$.

Perrineau, P. (1997) Le symptôme Le Pen, Paris, Fayard.

--- (2005), “Does Lepenism exist without Le Pen?” en: Casals, X., Political survival on the extreme right. European movements between the inherited past and the need to adapt to the future, Barcelona: Institut de Ciències Polítiques i Socials

Pont, A. y Solís, D. (2009) "Abstenció i desafecció", Revista de debat politic, Barcelona: Fundació Rafael Campalans

Rordríguez, J. L. (2006) "De la vieja a la nueva extrema derecha (pasando por la fascinación por el fascismo)”, Historia Actual Online, 9, descargado el 20/01/08.

--- (2010) "The Spanish extreme right: from Neo-Francoism to xenophobic discourse", en "Mapping the extreme right in contemporary Europe: from local to transnational” (eds.) Mammone, A.; Godin, E. y Jenkins, B., Oxford, Berghahn Books.

Rydgren, J. (2005) "Is extreme right-wing populism contagious? Explaining the emergence of a new party family", European Journal of Political Research, (44): 413-37

--- (2007) "The sociology of the radical right", Annual Review of Sociology 33: 241-62. 
Taguieff, P. A. (2007) "Interpretar la ola populista en la Europa contemporánea: entre resurgencia y emergencia”, en: Simón, M.A., La extrema derecha en Europa desde 1945 a nuestros días, Madrid: Tecnos

Storm, I. (2011) "Christian nations? Ethnic christianity and anti-immigration attitudes in four western european countries", Nordic Journal of Religion and Society, 24 (1): 75-96

Swyngedouw, Marc (2001) "The subjective cognitive and affective map of extreme right voters: using open-ended questions in open-ended questions in exit polls", Electoral studies, 20: 217-241

Vallès, J. M. (2008) (coord.) Actituds polítiques i comportament electoral a Catalunya: materials per un debat social, Barcelona: Direcció General de Participació Ciutadana-Generalitat de Catalunya.

Zúquete, J. P. (2008) "The European extreme-right and Islam: New directions?” Journal of Political Ideologies, 13 (3): 321-344 